\title{
BILINEAR FORMS AND 2-DIMENSIONAL COHOMOLOGY
}

\author{
MARTIN MOSKOWITZ \\ (Received 2 March 1984; revised 11 November 1984) \\ Communicated by Gavin Brown
}

\begin{abstract}
This paper calculates the central Borel 2 cocycles $Z_{\text {cent }}^{2}(G, A)$ for certain 2-step nilpotent Lie groups $G$ with values in the injectives $A$ of the category of 2 nd countable locally compact abelian groups. The $G$ 's include, among others, all groups locally isomorphic to a Heisenberg group. The $A$ 's are direct sums of vector groups and (possibly infinite dimensional) tori, and in particular include $\mathbb{R}, \mathbb{T}$, and $\mathbb{C}^{x}$. The main results are as follows.

(4.1) Every symmetric central 2 cocycle is trivial.

(4.2) Every central 2 cocycle is cohomologous with a skew symmetric bimultiplicative one (which is necessarily jointly continuous by [7]).

(4.3) The corresponding cohomology group $H_{\text {cent }}^{2}(G, A)$ is calculated as the skew symmetric jointly continuous bimultiplicative maps modulo $\operatorname{Hom}_{\text {cont }}\left([G, G]^{-}, A\right)$.

These results generalize the case when $G$ is a connected abelian Lie group and $A=\mathbf{T}$, due to Kleppner [3]. Using standard facts of the cohomology of groups they can be interpreted as classifying all continuous central extensions

$$
\text { (1) } \rightarrow A \rightarrow E \rightarrow G \rightarrow(1)
$$

of the group $G$ by the abelian group $A$. Finally some counterexamples are given to extending these results.
\end{abstract}

1980 Mathematics subject classification (Amer. Math. Soc.): 22 E 41.

\section{Introduction}

In studying representations of locally compact groups, projective representations arise as a natural and inescapable generalization. It is therefore of interest to determine all possible multipliers or central 2-cocycles on the group with values in

(C) 1986 Australian Mathematical Society $0263-6115 / 86 \$ A 2.00+0.00$ 
the circle if one is interested in unitary representations or the non-zero complex numbers in general. Of course structural considerations are another important motivating factor; namely, one wishes to find all central extensions of a given group $G$ by a given abelian group $A$. In this paper we shall essentially determine all possible central 2 cocycles for a certain class of non-abelian groups $G$ with values in the class of injectives $A$ for the category of locally compact abelian groups (Theorem 4.2). Then we shall compute the associated cohomology group (Theorem 4.3). The $A$ 's are direct sums of vector groups and (possibly infinite dimensional) tori. The $G$ 's will be certain 2-step nilpotent groups (those satisfying * of Section 2). These include, among others, all groups which are locally isomorphic to a Heisenberg group. The notions of a bimultiplicative map, symmetry, skew symmetry, 2 cocycle and $H_{\text {cent }}^{2}(G, A)$ are defined in Section 1.

Our main results are as follows.

(4.2) Every central 2 cocycle is cohomologous with a skew symmetric bimultiplicative one.

(4.3). $\quad H_{\text {cent }}^{2}(G, A)=$ skew symmetric bimultiplicative maps modulo $\operatorname{Hom}_{\text {cont }}\left([G, G]^{-}, A\right)$.

\section{Preliminaries}

Let $G$ and $A$ be second countable locally compact groups written multiplicatively, with $A$ abelian. Further properties of $G$ and $A$ will be specified later. We shall be concerned with Borel measurable functions $\omega: G \times G \rightarrow A$ satisfying $\omega(1, x)=1=\omega(x, 1)$ for all $x \in G$ and $\omega(x y, z) \omega(x, y)=\omega(x, y z) \omega(y, z)$ for all $x, y, z \in G$, henceforth called 2 cocycles. The set of all 2 cocycles is denoted $Z^{2}(G, A)$. It is an abelian group under pointwise operations. An easy calculation shows that examples of 2 cocycles are provided as follows. Let $f: G \rightarrow A$ be a measurable function, and let $\omega_{f}(x, y)=f(x) f(y) f(x y)^{-1}$ for $x, y \in G$. It is easy to see that $\omega_{f} \omega_{f^{\prime}}=\omega_{f f^{\prime}}$. Two cocycles $\omega$ and $\omega^{\prime}$ are called cohomologous if $\omega=\omega^{\prime} \omega_{f}$ for some $f$. So if $\omega$ is cohomologous with $\omega_{f}$, then it equals $\omega_{f^{\prime}}$ for some $f^{\prime}$, and we say that $\omega$ is trivial. Also the set $B^{2}(G, A)$ of trivial cocycles is a subgroup, and by definition $H_{\text {cent }}^{2}(G, A)=Z^{2}(G, A) / B^{2}(G, A)$.

Important facts about central 2 cocycles are the following (see G. Mackey [4] and C. C. Moore [5]). Given a 2 cocycle $\omega$, one gets a topological group extension

$$
\text { (1) } \rightarrow A \rightarrow E_{\omega} \rightarrow G \rightarrow(1),
$$

where $A$ is a closed central subgroup and $E / A \cong G$. Here $E_{\omega}=G \times A$ as a set, and $(a, x)\left(a^{\prime}, x^{\prime}\right)=\left(a a^{\prime} \omega\left(x, x^{\prime}\right), x x^{\prime}\right)$. Now $A$ and $G$ have natural Borel structures which are standard. The direct product defines a standard Borel structure 
on $E_{\omega}$, and the map $\left(e, e^{\prime}\right) \mapsto e e^{\prime-1}$ is measurable, so that $E_{\omega}$ is a standard Borel group. Since the direct product of the Haar measures is an invariant measure on $E_{\omega}$, it follows by (7.1) of [4] that $E_{\omega}$ has a unique locally compact topology in which it is a separable group with the above Borel structure. Two such extensions are equivalent in the obvious sense of the diagram below

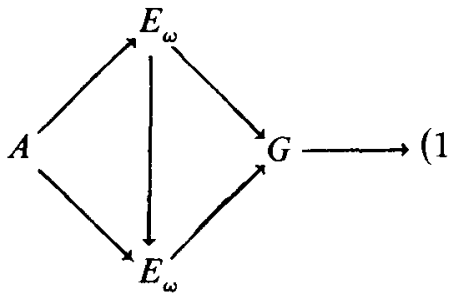

if and only if $\omega$ and $\omega^{\prime}$ are cohomologous [5].

A well known but interesting example of a 2 cocycle is provided by the following. Let $\beta: G \times G \rightarrow A$ be measurable and multiplicative in each variable separately. Then $\beta$ is easily seen to be a cocycle. We denote by $B_{A}(G, G)$ the set of such cocycles. It is easily seen to be a subgroup of $Z^{2}(G, A)$. It is the purpose of this paper to explore the relationship between $B_{A}(G, G)$ (in fact the skew symmetric part of $B_{A}(G, G)$ ) and all of $Z^{2}(G, A)$, that is to investigate under what circumstances a general 2 cocycle is cohomologous with a bilinear one. Our assumption on $A$ beginning in Section 4 will be that $A$ is an injective for the category of locally compact abelian groups (see [6], where the projectives and injectives of this category are computed). Because of second countability, this means that $A=\mathbb{R}^{n} \times \mathbb{T}^{*}$, where $\boldsymbol{\kappa} \leqslant \boldsymbol{N}_{0}$, and where $\mathbb{R}$ and $\mathbb{T}$ are, respectively, the reals and reals mod 1 . $A$ 's of special interest of course are $\mathbb{T}, \mathbb{R}_{+}^{x}(=\mathbb{R})$ and their direct sum $\mathbb{T} \times \mathbb{R}_{+}^{x}=\mathbf{C}^{x}$. By [7], the elements of $B_{A}(G, G)$ are actually jointly continuous if, for instance, $G$ is a connected Lie group and $A$ is as above. Notice that the well known Heisenberg group $N_{r}$ (of dimension $2 r+1$ ), $r \geqslant 1$, is given by such an extension

$$
(1) \rightarrow \mathbb{R} \rightarrow N_{r} \rightarrow \mathbb{R}^{2 r} \rightarrow(1),
$$

and in fact the multiplication is given in additive notation as follows:

$$
(z, X, Y)\left(z^{\prime}, X^{\prime}, Y^{\prime}\right)=\left(z+z^{\prime}+\left\langle X, Y^{\prime}\right\rangle, X+X^{\prime}, Y+Y^{\prime}\right) .
$$

Thus, $\omega\left((X, Y),\left(X^{\prime}, Y^{\prime}\right)\right)=\left\langle X, Y^{\prime}\right\rangle$ (inner product in $\left.\mathbb{R}^{r}\right)$ is bilinear. This is not an accident, since, for $\mathbf{T}$ valued cocycles, such results were proven by A. Kleppner [3] if, for example, $G$ is abelian and simply connected (the hypothesis used in [3] is that each element of $G$ must have a unique square root). Simple modifications in this situation carry over to $\mathbb{R}$ valued cocycles and indeed $A$-valued ones, with 
$A$ as above. The objective here is to investigate the question for non-abelian groups $G$.

\section{Abelianization and decomposition into symmetric and skew symmetric parts}

For a topological group $G$, let $[G, G]$ be the derived group, $[G, G]^{-}$its closure, and $\pi$ the canonical map: $G \rightarrow G /[G, G]^{-}$.

Lemma 1.1. Let $\beta \in B_{A}(G, G)$, where $G$ and $A$ are $\sigma$-compact locally compact groups. Then there exists $\beta^{*} \in B_{A}\left(G /[G, G]^{-}, G /[G, G]^{-}\right)$such that $\beta=$ $\beta^{*} \circ(\pi, \pi)$.

Proof. Suppose $\bar{x}=\bar{y}$ and $\bar{z}=\bar{w}$. Then $x=y c$ and $z=w d$, where $x, y, z, w$ $\in G$, and $c, d \in[G, G]^{-}$. Then

$$
\beta(x, z)=\beta(y c, w d)=\beta(y, w) \beta(c, w) \beta(y, d) \beta(c, d) .
$$

Now by multiplicativity and the fact that $A$ is abelian, $\beta(y, d)=1$ for $d \in[G, G]$. Since $\beta$ is measurable, it is measurable in the second variable. But it is multiplicative, and since $G$ and $A$ are $\sigma$-compact, it is continuous in the second variable (see [7]). Hence, $\beta(y, d)=1$ for all $d \in[G, G]^{-}$. This shows the last two terms are each equal to 1 . Similarly $\beta(c, w)=1$. Thus $\beta(x, z)=\beta(y, w)$. Clearly $\beta^{*}(\pi, \pi)=\beta$ and $\beta^{*} \in B_{A}\left(G /[G, G]^{-}, G /[G, G]^{-}\right)$because $\beta$ is bimultiplicative, $(\pi, \pi)$ is open, and open sets generate the $\sigma$-algebra of Borel sets.

Definition. If $\omega \in Z^{2}(G, A)$, we shall write $\omega^{\sim}(x, y)=\omega(y, x)$. We shall call $\omega$ symmetric if $\omega=\omega \sim$ and skew symmetric if $\omega^{-1}=\omega \sim$. The symmetric and skew symmetric parts of $B_{A}(G, G)$ will be denoted by $B_{A}(G, G)_{s}$ and $B_{A}(G, G)_{a}$, respectively.

LEMMA 1.2. $\left(\omega^{-1}\right)^{\sim}=\left(\omega^{\sim}\right)^{-1}$ for all $\omega \in Z^{2}(G, A)$; moreover, if $\omega \in Z^{2}(G, A)$, then so does $\omega$.

Proof. $\left(\omega^{-1}\right)^{\sim}(x, y) \omega \sim(x, y)=\omega^{-1}(y, x) \omega(y, x)=1$, so $\left(\omega^{-1}\right) \omega^{\sim}=1$. Now if $\omega \in Z^{2}(G, A)$, then $\omega \sim(x, 1)=\omega(1, x)=1$, and $\omega \sim(1, x)=\omega(x, 1)=1$. Also $\omega(z, x y) \omega(y, x)=\omega(y z, x) \omega(z, y)$ for all $x, y, z \in G$. Consider the bijective map $t \rightarrow t^{-1}$ of $G$. Then the latter yields $\omega\left(z^{-1}, x^{-1} y^{-1}\right) \omega\left(y^{-1}, x^{-1}\right)=$ $\omega\left(y^{-1} z^{-1}, x^{-1}\right) \omega\left(z^{-1}, y^{-1}\right)$. But $\omega\left(z^{-1} y, x^{-1} y^{-1}\right)=\omega\left(z^{-1},(y x)^{-1}\right)$, and $\omega\left(y^{-1} z^{-1}, x^{-1}\right)=\omega\left((z y)^{-1}, x^{-1}\right)$. Substituting and applying $t \rightarrow t^{-1}$ again yields the identity $\omega(z, y x) \omega(y, x)=\omega(z y, x) \omega(z, y)$. This is the cocycle identity for $\omega$.

The point here of reproving the important Lemma (1.3) presented in [3] is that now it holds for non-abelian but $\sigma$-compact groups $G$ and $A$. 
Lemma 1.3. If $\omega \in Z^{1}(G, A)$, then $\omega(\tilde{\omega})^{-1} \in B_{A}(G, G)_{a}$. Moreover, (by [7]) it is jointly continuous.

Proof. $\omega\left(\omega^{\sim}\right)^{-1}(x y, z)=\omega(x y, z)\left(\omega^{-1}\right)^{\sim}(x y, z)=\omega(x y, z) \omega^{-1}(z, x y)=$ $\omega(x y, z) \omega(x, y) \omega(x, y)^{-1} \omega(z, x y)^{-1}$. By the cocyle identity the latter equals

$$
\begin{aligned}
& \omega(x, y z) \omega(y, z) \omega(x, y)^{-1} \omega(z, x y)^{-1} \\
& \quad=\omega(x, y z) \omega(y, z)(\omega(z, x) \omega(z x, y))^{-1} \\
& =\omega(x, y z) \omega(y, z) \omega(x, z) \omega(x, z)^{-1} \omega(z, x)^{-1} \omega(z x, y)^{-1} .
\end{aligned}
$$

Does this equal

$$
\omega\left(\omega^{\sim}\right)^{-1}(x, z) \omega\left(\omega^{\sim}\right)^{-1}(y, z),
$$

i.e. $\omega(x, z) \omega(z, x)^{-1} \omega(y, z) \omega(z, y)^{-1}$ ? If we cancel out common factors this question becomes "Does $\omega(x, y z) \omega(z, y)=\omega(x, z) \omega(z x, y)$ ?" Since $\omega \in$ $Z^{2}(G, A), \omega^{-}$is also a cocycle by (1.2). In terms of $\omega^{\sim}$ this says that $\omega \sim(y, z) \omega \sim(y z, x)=\omega \sim(z, x) \omega \sim(y, z x)$, which proves the statement above.

Now $\omega\left(\omega^{\sim}\right)^{-1}$ is multiplicative in the left variable. But $\left.\omega^{(} \omega^{\sim}\right)^{-1} \omega^{-1} \omega^{\sim}=$ $\omega \omega^{-1}\left(\omega^{\sim}\right)^{-1} \omega^{\sim}=1$. So $\left(\omega^{\prime}\left(\omega^{\sim}\right)^{-1}\right)^{-1}=\omega^{-1} \omega^{\sim}$. Clearly $\left(\omega\left(\omega^{\sim}\right)^{-1}\right)^{\sim}=\omega^{\sim} \omega^{-1}=\omega^{-1} \omega^{\sim}$, since $Z^{2}(G, A)$ is abelian. Thus $\omega\left(\omega^{\sim}\right)^{-1}$ is skew symmetric. Therefore it is also multiplicative in the right variable.

Lemma 1.4. Let $G /[G, G]^{-}$be a connected and simply connected Lie group. If $\beta \in B_{A}(G, G)$, then there exists $\gamma \in B_{A}(G, G)$ with $\gamma^{2}=\beta$.

Proof. Case 1. $G$ is an abelian group and each element has a unique square root (which is a measurable function). When $A=\pi$, this is Kleppner's case. For completeness we furnish the simple argument. Let $(g, h) \in G \times G$. Then $x^{2}=g$ for a unique $x \in G$. Define $\gamma(g, h)=\beta(x, h)$. If $x_{1}^{2}=g_{1}$ and $x_{2}^{2}=g_{2}$, then $\left(x_{1} x_{2}\right)^{2}=x_{1}^{2} x_{2}^{2}=g_{1} g_{2}$, and so $\gamma\left(g_{1} g_{2}, h\right)=\beta\left(x_{1} x_{2}, h\right)$ and $\gamma\left(g_{1}, h\right) \gamma\left(g_{2}, h\right)=$ $\beta\left(x_{1}, h\right) \beta\left(x_{2}, h\right)$. Since $\beta$ is multiplicative, so is $\gamma$. Now $\gamma \sim(g, h)=\gamma(h, g)=$ $\beta(y, g)$, where $y^{2}=h$, and $\gamma(g, h)=\beta(x, h)$, where $x^{2}=g$. So $\gamma \sim(g, h) \gamma(g, h)$ $=\beta\left(y, x^{2}\right) \beta\left(x, y^{2}\right)=\beta(y, x)^{2} \beta(x, y)^{2}=(\beta(y, x) \beta(x, y))^{2}=1^{2}=1$, since $\beta$ is skew symmetric. Thus, $\gamma$ is skew symmetric. Since it is multiplicative in the left variable, it is therefore multiplicative in the right variable. Finally, $\gamma^{2}(g, h)=$ $\gamma(g, h)^{2}=\beta(x, h)^{2}=\beta\left(x^{2}, h\right)=\beta(g, h)$ so that $\gamma^{2}=\beta$. Since $\beta$ is measurable, so is $\gamma$.

Case 2. $G /[G, G]$ is connected and simply connected and each element has a unique square root. Form $\beta^{*}$ as in (1.1). Now $\beta^{*}(\bar{x}, \bar{y})=\beta(x, y)$, and $\beta^{*}(\bar{y}, \bar{x})$ $=\beta(y, x)$, so $\beta^{*}(\bar{x}, \bar{y}) \beta^{*}(\bar{y}, \bar{x})=\beta(x, y) \beta(y, x)=1$. Thus $\beta^{*}$ is skew symmet- 
ric. By Case 1 there exists $\gamma \in B_{A}\left(G /[G, G]^{-}, G /[G, G]^{-}\right)_{a}$ such that $\gamma^{2}=\beta^{*}$. Let $\gamma_{1}$ be $\gamma$ lifted to $G \times G$; that is, $\gamma_{1}(x, y)=\gamma(\bar{x}, \bar{y})$. Then clearly $\gamma_{1}$ is skew symmetric and measurable, since $\gamma$ and $(\pi, \pi)$ are measurable. Since $\pi$ is a homomorphism, we see that $\gamma_{1} \in B_{A}(G, G)$. Now $\gamma_{1}^{2}(x, y)=\gamma(\bar{x}, \bar{y})^{2}=\beta(\bar{x}, \bar{y})$ $=\beta(x, y)$.

COROllary. 1.5. If $\omega \in Z^{2}(G, A)$, then there exists $\gamma \in B_{A}(G, G)_{a}$ with $\gamma^{2}=$ $\omega\left(\omega^{-}\right)^{-1}$ (this follows from (1.3) and (1.4)). In particular, $\gamma^{2}=\beta\left(\beta^{\sim}\right)^{-1}$ for any $\beta \in B_{A}(G, G)$.

\section{Some structural results}

Our initial assumption on the second countable locally compact group $G$ is the following: there exist closed abelian subgroups $H_{1}$ and $K_{1}$ of $G$ such that $G=H_{1} K_{1}$ and $H_{1} \cap K_{1} \supseteq Z(G)$, the center of $G$. We shall provisionally call this condition $C$.

LEMMA 2.1. $H_{1}$ and $K_{1}$ are normal if and only if $G$ is 2-step nilpotent.

Proof. If $H_{1}$ and $K_{1}$ are normal, then $H_{1} \cap K_{1}$ is closed, and we get an injective homomorphism $G /\left(H_{1} \cap K_{1}\right) \rightarrow G / H_{1} \times G / K_{1}$. Now $G / H_{1}=$ $H_{1} K_{1} / H_{1} \cong K_{1} /\left(H_{1} \cap K_{1}\right)$, which is abelian since $K_{1}$ is. Similarly $G / K_{1}$ is also abelian. This means that $G /\left(H_{1} \cap K_{1}\right)$, hence also $G / Z(G)$, abelian. Conversely, if $G$ is 2-step nilpotent, then $\left[H_{1}, G\right] \subseteq[G, G] \subseteq Z(G) \subseteq H_{1}$. So $H_{1}$, and similarly $K_{1}$, are normal.

Condition $C$ together with the normality of $H_{1}$ and $K_{1}$ will constitute our basic assumption about $G$. This we shall call *

COROLlaRY 2.2. If $G$ is a connected Lie group satisfying *, then, in particular, $G$ is nilpotent, so the exponential map is onto, and each $g \in G$ has a square root. If $G /[G, G]^{-}$is connected and simply connected, then each element of it has a unique square root. In particular, if $G$ is connected and simply connected, then this is so (since $[G, G]^{-}$is connected).

LEMMA 2.3. If $G$ is simply connected, then * is equivalent to the following. Let $\mathfrak{g}$ be the 2-step nilpotent Lie algebra of $G$. Then one can choose a basis $\left\{Z_{1}, \ldots, Z_{s}\right\}$ of $z(g)$ and extend this to a basis $\left\{Z_{1}, \ldots, Z_{s}, X_{1}, \ldots, X_{n}, Y_{1}, \ldots, Y_{m}\right\}$ of $\mathfrak{g}$ so that $\left[X_{i}, X_{j}\right]=0=\left[Y_{k}, Y_{l}\right]$ for all $i, j=1, \ldots, n$ and $k, l=1, \ldots, m$.

Proof. Let $\mathfrak{h}_{1}=1 s_{\mathbf{R}}\left\{Z_{1}, \ldots, Z_{s}, X_{1}, \ldots, X_{n}\right\}$, and let $\mathfrak{f}_{1}=$ $1 s_{\mathbf{R}}\left\{Z_{1}, \ldots, Z_{s}, Y_{1}, \ldots, Y_{m}\right\}$. Then $\mathfrak{h}_{1}$ and $\mathfrak{f}_{1}$ are abelian subalgebras, $\mathfrak{h}_{1}+\mathfrak{f}_{1}=\mathfrak{g}$, 
and $\mathfrak{h}_{1} \cap \mathfrak{f}_{1}=\mathfrak{z}(\mathfrak{g})$. Now $\left[\mathfrak{h}_{1}, \mathfrak{f}_{1}\right] \subseteq[\mathfrak{g}, \mathfrak{g}] \subseteq \mathfrak{z}(\mathfrak{g})=\mathfrak{h}_{1} \cap \mathfrak{f}_{1}$, so $\mathfrak{h}_{1}$ and $\mathfrak{f}_{1}$ are ideals. Let $H_{1}$ and $K_{1}$ be the corresponding normal analytic subgroups. Then $H_{1}$ and $K_{1}$ are abelian, $H_{1} \cap K_{1}=Z(G)$, and $H_{1}$ and $K_{1}$ closed because $G$ is simply connected. If $g=\exp X \in G$, then $X=h_{1}+k_{1}$, where $h_{1} \in \mathfrak{h}_{1}, k_{1} \in \mathfrak{f}_{1}$. But $\exp h_{1} \exp k_{1}=\exp \left(h_{1}+k_{1}+\frac{1}{2}\left[h_{1}, k_{1}\right]\right)$ by the Campbell Hausdorff Formula. Since $\frac{1}{2}\left[h_{1}, k_{1}\right] \in z(\mathfrak{g})$, the latter equals $\exp \left(h_{1}+k_{1}\right) \exp \left(\frac{1}{2}\left[h_{1}, k_{1}\right]\right)$, and so $g=\exp h_{1} \exp k_{1} \exp \left(-\frac{1}{2}\left[h_{1}, k_{1}\right]\right)$. Since $\left[\mathfrak{h}_{1}, \mathfrak{f}_{1}\right] \subseteq \mathfrak{f}_{1}$, the product of the latter two factors is in $K_{1}$, so $g \in H_{1} K_{1}$. Conversely, if $G$ has property *, then the ideals $\mathfrak{h}_{1}$ and $\mathfrak{f}_{1}$ corresponding to $H_{1}$ and $K_{1}$ are abelian, and $\mathfrak{h}_{1} \cap \mathfrak{f}_{1} \supseteq \mathfrak{z}(\mathfrak{g})$. If $x \in \mathrm{g}$, and if $g=\exp X=\exp h_{1} \exp k_{1}$, then, by *, and by the fact that exp is surjective for $\mathfrak{h}_{1}$ and $\mathfrak{f}_{1}$, we see from the Campbell Hausdorff Formula that $X=\log \left(\exp h_{1} \exp k_{1}\right)=h_{1}+k_{1}+\frac{1}{2}\left[h_{1}, k_{1}\right]$. Since $\mathfrak{f}_{1}$ is an ideal, $\mathfrak{g}=\mathfrak{h}_{1}+\mathfrak{f}_{1}$.

(2.4). An example is now clearly provided by the Heisenberg group $N_{r}$. Let $\mathfrak{n}_{r}$ be its Lie algebra. Then $\mathfrak{n}_{r}$ is generated by $X=\left(x_{1}, \ldots, x_{r}\right)$, by $Y=\left(y_{1}, \ldots, y_{r}\right)$ $\in R^{r}$, and by $z \in \mathbb{R}$, with bracketing $\left[(X, Y, z),\left(X^{\prime}, Y^{\prime}, z^{\prime}\right)\right]=\left(0,0,\left\langle Y, X^{\prime}\right\rangle-\right.$ $\left.\left\langle X, Y^{\prime}\right\rangle\right)$. Clearly then $\mathfrak{h}_{1}=\{(X, 0, z)\}$ and $\mathfrak{f}_{1}=\{(0, Y, z)\}$ are abelian ideals with $\mathfrak{h}_{1}+\mathfrak{f}_{1}=\mathfrak{g}$ and $\mathfrak{h}_{1} \cap \mathfrak{f}_{1}=\mathfrak{z}(\mathfrak{g})$. By (2.3), * is satisfied here. More generally, let $g$ be any 2-step nilpotent Lie algebra, and let $\left\{Z_{1}, \ldots, Z_{s}\right\}$ be a basis of $z(g)$. Extend this to a basis $\left\{Z_{1}, \ldots, Z_{s}, X_{1}, \ldots, X_{n}\right\}$ of $\mathfrak{g}$. Then

(i) $\left[X_{i}, X_{j}\right]=\sum_{k=1}^{s} C_{i, j}^{k} Z_{k}$, where $c_{i, j}^{k} \in \mathbb{R}$ and $i, j=1, \ldots, n$; and

(ii) all other brackets among $\left\{Z_{1}, \ldots, Z_{s}, X_{1}, \ldots, X_{n}\right\}$ are zero except for $\left[X_{j}, X_{i}\right]=-\left[X_{i}, X_{j}\right]$.

Conversely, if $\left\{Z_{1}, \ldots, Z_{s}, X_{1}, \ldots, X_{n}\right\}$ is an independent over $\mathbb{R}$ set of elements in a vector space $g$, and if bracketing is defined by (i) and (ii), then $\left[Z_{i}, X\right]=0$ for all $X \in \mathfrak{g}$ and $i=1, \ldots, s$. Hence any triple bracket is 0 . Therefore the Jacobi identity is automatically satisfied, and since

$$
[\mathfrak{g}, \mathfrak{g}] \subseteq 1 s_{\mathbf{R}}\left\{Z_{1}, \ldots, Z_{s}\right\} \subseteq \mathfrak{z}(\mathfrak{g})
$$

it follows that $\mathrm{g}$ is 2-step nilpotent.

Now by (2.3), * (or rather the corresponding Lie algebra condition) is satisfied by $g$ if and only if we can partition $\left\{X_{1}, \ldots, X_{n}\right\}$ into $\left\{X_{1}, \ldots, X_{t}, X_{t+1}, \ldots, X_{n}\right\}$ so that $\left[X_{i}, X_{j}\right]=0$ if $i, j \leqslant t$ or $i, j \geqslant t+1$. Since $Z_{1}, \ldots, Z_{s}$ are linearly independent, this holds if and only if $c_{i, j}^{k}=0$ for $i, j \leqslant t$ or $i, j \geqslant t+1$. In other words, the $c_{i, j}^{k}$ must form a family of $s, t \times n-t$ matrices. In this case we would take $\mathfrak{h}_{1}=1 s_{\mathbf{R}}\left\{Z_{1}, \ldots, Z_{s}, X_{1}, \ldots, X_{t}\right\}$ and $\mathfrak{f}_{1}=1 s_{\mathbf{R}}\left\{Z_{1}, \ldots, Z_{s}, X_{t+1}, \ldots, X_{n}\right\}$ and argue as in (2.3). Clearly such a partition need not exist, so our results need not apply even to arbitrary 2-step nilpotent groups. But, for example, if $\operatorname{dim}=4$, then by the above together with (2.5) below, * is always satisfied.

Some sufficient conditions, and a characterization of $\mathfrak{n}_{r}$, follow. 
Proposition 2.5. Let $\mathfrak{g}$ be a 2-step nilpotent Lie algebra over $\mathbb{R}$ with 1-dimensional center. Then $\mathfrak{g} \cong \mathfrak{n}_{r}$ for some $r$. In particular, * is satisfied.

Proof. $[\mathfrak{g}, \mathfrak{g}]=\mathfrak{z}(\mathfrak{g})$. If we let $\beta(X, Y)=[X, Y]$, then $\beta: \mathfrak{g} \times \mathfrak{g} \rightarrow[\mathfrak{g}, \mathfrak{g}]$ is a skew symmetric bilinear form. If either $X$ or $Y$ belongs to $z(g)$, then $\beta(X, Y)=0$. Hence $\beta$ induces a skew symmetric bilinear form: $\mathfrak{g} / \mathfrak{z}(\mathfrak{g}) \times \mathfrak{g} / \mathfrak{z}(\mathfrak{g}) \rightarrow[\mathfrak{g}, \mathfrak{g}]$. Now if $\beta(X+z, Y+z)=0$ for all $Y \in \mathfrak{g}$, then $[X, Y]=0$ for all $Y$, so that $X \in z$ and $X+z=\overline{0}$. Thus the form is of maximal rank, and $\operatorname{dim}(g / z(g))$ is even, say equal to $2 r$, by skew symmetry. Thus $\operatorname{dim} \mathfrak{g}=2 r+1$, and $\left[(X, Y, z),\left(X^{\prime}, Y^{\prime}, z^{\prime}\right)\right]$ for all $(X, Y, z)$, and $\left(X^{\prime}, Y^{\prime}, z^{\prime}\right) \in \mathfrak{g}$, is defined by

$$
\left(A\left(\begin{array}{c}
x_{1} \\
y_{1} \\
\vdots \\
x_{r} \\
y_{r}
\end{array}\right),\left(\begin{array}{c}
x_{1}^{\prime} \\
y_{1}^{\prime} \\
\vdots \\
x_{r}^{\prime} \\
y_{r}^{\prime}
\end{array}\right)\right),
$$

where (, ) is the usual inner product on $\mathbb{R}^{2 r}, X=\left(x_{1}, \ldots, x_{r}\right), Y=\left(y_{1}, \ldots, y_{r}\right)$, $X^{\prime}=\left(x_{1}^{\prime}, \ldots, x_{r}^{\prime}\right), Y^{\prime}=\left(y_{1}^{\prime}, \ldots, y_{r}^{\prime}\right)$, and where

$$
A=\left(\begin{array}{rrrr}
0 & -1 & 0 & 0 \\
1 & 0 & 0 & 0 \\
0 & 0 & 0 & -1 \\
0 & 0 & 1 & 0
\end{array}\right)
$$

is a $2 r$ by $2 r$ matrix. Direct calculation tells us that $\left[(X, Y, z),\left(X^{\prime}, Y^{\prime}, z^{\prime}\right)\right]=$ $\left\langle X, Y^{\prime}\right\rangle-\left\langle Y, X^{\prime}\right\rangle$, where $\langle$,$\rangle is the usual inner product on \mathbb{R}^{r}$. Therefore, $\left[(X, 0, o),\left(X^{\prime}, 0, o\right)\right]=0=\left[(0, Y, o),\left(0, Y^{\prime}, o\right)\right]$. So $\left\{(X, 0, o): \quad X \in \mathbb{R}^{r}\right\}$ and $\left\{(0, Y, o): Y \in \mathbb{R}^{r}\right\}$ are abelian subalgebras, $\{(0,0, z): z \in \mathbb{R}\}$ is the center, and $[(X, 0, o),(0, Y, o)]=\langle X, Y\rangle$. Thus $\mathfrak{g} \cong \mathfrak{n}_{r}$.

Let $G, H$ and $A$ be locally compact abelian groups and $\beta$ : $G \times H \rightarrow A$ a bimultiplicative map. This gives rise to a bimultiplicative (polarized) map $\omega$ : $(G \times H) \times(G \times H) \rightarrow A$ as follows: $\omega\left((g, h),\left(g^{\prime}, h^{\prime}\right)\right) \in \beta\left(g, h^{\prime}\right)$. We form $E$ $=E_{\omega}$ as usual and get a central extension.

$$
\text { (1) } \rightarrow A \rightarrow E \stackrel{\pi}{\rightarrow} G \times H \rightarrow \text { (1), }
$$

where multiplication is given by $(a, g, h)\left(a^{\prime}, g^{\prime}, h^{\prime}\right)=\left(a a^{\prime} \beta\left(g, h^{\prime}\right), g g^{\prime}, h h^{\prime}\right)$. Now since $\omega\left((g, 1),\left(g^{\prime}, 1\right)\right)=\beta(g, 1)=1$ and $\omega\left((1, h),\left(1, h^{\prime}\right)\right)=\beta\left(1, h^{\prime}\right)=1$, we see that the closed normal subgroups $\pi^{-1}(G)$ and $\pi^{-1}(H)$ of $E$ are abelian. Also $\pi^{-1}(G) \cap \pi^{-1}(H) \supseteq A$ and $\pi^{-1}(G) \pi^{-1}(H)=E$. Now if $\beta$ is nondegenerate, then $A=Z(E)$, so that $\pi^{-1}(G) \cap \pi^{-1}(H) \supset Z(E)$. 
An easy calculation shows that

$$
\left[(a, g, h),\left(a^{\prime}, g^{\prime}, h^{\prime}\right)\right]=\left(\beta\left(g^{\prime}, h\right)^{-1}, 1,1\right) .
$$

Hence if $\beta$ has dense range, then $[E, E]^{-}=A$ and $E /[E, E]^{-}=G \times H$. Thus we have another class of examples

Proposition 2.6. Let $G, H$, and $A$ be locally compact abelian groups, and let $\beta$ : $G \times H \rightarrow A$ be a bimultiplicative map. Suppose that

(i) $\beta: G \times H \rightarrow A$ is a nondegenerate form with dense range,

(ii) $G$ and $H$ have unique square roots.

Then $E$ satisfies * and $E /[E / E]$ has unique square roots.

\section{Symmetric cocycles}

Let $\omega: G \times G \rightarrow A$ be a Borel cocycle which is symmetric.

LEMMA 3.1. $\omega\left(g g^{\prime},\left(g^{\prime} g\right)^{-1}\right)=\omega\left(g^{\prime} g,\left(g^{\prime} g\right)^{-1}\right)$ for $g, g^{\prime} \in G$.

Proof. Using the central cocycle identity, we get

$$
\omega\left(g g^{\prime},\left(g^{\prime} g\right)^{-1}\right) \omega\left(1, g^{\prime \prime}\right)=\omega\left(\left(g^{\prime} g\right)^{-1}, g^{\prime \prime}\right) \omega\left(g g^{\prime},\left(g^{\prime} g\right)^{-1} g^{\prime \prime}\right)
$$

Since $\omega\left(1, g^{\prime \prime}\right)=1$ for all $g^{\prime \prime}$, it follows that if we take $g^{\prime \prime}=g^{\prime} g$, then we get

$$
\omega\left(g g^{\prime},\left(g^{\prime} g\right)^{-1}\right)=\omega\left(\left(g^{\prime} g\right)^{-1}, g^{\prime} g\right) \omega\left(g g^{\prime}, 1\right) \text {. }
$$

The fact that the latter term is 1 and that $\omega$ is symmetric yields the result.

Corollary 3.2. If $\omega$ is symmetric, then in $E_{\omega}$ we have

$$
\left[(a, g),\left(a^{\prime}, g^{\prime}\right)\right]=\left(1,\left[g, g^{\prime}\right]\right) \text {. }
$$

Proof. We have

$$
\begin{aligned}
& {\left[(a, g),\left(a^{\prime}, g^{\prime}\right)\right]=(a, g)\left(a^{\prime}, g^{\prime}\right)\left(\left(a^{\prime}, g^{\prime}\right)(a, g)\right)^{-1}} \\
& \quad=\left(a a^{\prime} \omega\left(g, g^{\prime}\right), g g^{\prime}\right)\left(a^{\prime} a \omega\left(g^{\prime}, g\right)^{-1} \omega\left(g^{\prime} g,\left(g^{\prime} g\right)^{-1}\right)^{-1},\left(g^{\prime} g\right)^{-1}\right) \\
& \left.\quad=\left(a a^{\prime} \omega\left(g, g^{\prime}\right) a^{\prime-1} a^{-1} \omega\left(g^{\prime}, g\right)^{-1} \omega\left(g^{\prime}, g\right),\left(g^{\prime} g\right)^{-1}\right)^{-1} \omega\left(g g^{\prime},\left(g^{\prime} g\right)^{-1}\right),\left[g, g^{\prime}\right]\right) \\
& \quad=\left(1,\left[g, g^{\prime}\right]\right)
\end{aligned}
$$

by the symmetry of $\omega$ together with (3.1). 
COROllary 3.3. If $\omega$ is symmetric, and if $G$ is nilpotent, then the index of nilpotence of $E$ equals that of $G$.

LEMMA 3.4. If $\omega$ is symmetric, and if $G$ and $A$ are separable, where $A$ is injective for the category of locally compact abelian groups, then for each closed abelian subgroup $H$ of $G, E_{H}$ is abelian and $\omega\left(h, h^{\prime}\right) \equiv 1$ for all $h, h^{\prime} \in H$.

Proof. Consider $\omega \mid H \times H$, which is a central Borel cocycle of $H$. Since $\omega \mid H \times H$ is symmetric, the central extension $E_{H}$ given by

$$
\text { (1) } \rightarrow A \rightarrow E_{H} \rightarrow H \rightarrow \text { (1) }
$$

is abelian. $E_{H}$ is locally compact and separable by Mackey's theorem [4] for measurable cocycles. Since $A$ is injective, this splits. Hence $(a, h)\left(a^{\prime}, h^{\prime}\right)=$ $\left(a a^{\prime} \omega\left(h, h^{\prime}\right), h h^{\prime}\right)=\left(a a^{\prime}, h h^{\prime}\right)$, and so $\omega\left(h, h^{\prime}\right)=1$.

Now take $H=Z(G)$, the center, and assume that $G$ satisfies *. Then $G$ is 2-step nilpotent by (2.1), so that $[G, G] \subseteq Z(G)$. Hence we get

Corollary 3.5. $\omega\left(c, c^{\prime}\right) \equiv 1$ for $c, c^{\prime} \in[G, G]$.

Now consider the extension $E_{\omega}=E$

$$
\text { (1) } \rightarrow A \rightarrow E \rightarrow G \rightarrow(1)
$$

for $\omega$ symmetric.

LEMMA 3.6. Let $x=\left[e_{1}, e_{1}^{\prime}\right] \cdots\left[e_{n}, e_{n}^{\prime}\right] \in[E, E]$, where $e_{i}=\left(a_{i}, g_{i}\right)$ and $e_{i}^{\prime}=$ $\left(a_{i}^{\prime}, g_{i}^{\prime}\right)$ are in $E$. Then $x=\left(1,\left[g_{1}, g_{1}^{\prime}\right] \cdots\left[g_{n}, g_{n}^{\prime}\right]\right)$.

Proof. By (3.2), $\left[e_{i}, e_{i}^{\prime}\right]=\left(1,\left[g_{i}, g_{i}^{\prime}\right]\right)$ for each $i$. Induction then yields $x=\left(1,\left[g_{1}, g_{1}^{\prime}\right] \cdots\left[g_{n-1}, g_{n-1}^{\prime}\right]\right)\left(1,\left[g_{n}, g_{n}^{\prime}\right]\right)=\left(\omega\left(\left[g_{1}, g_{1}^{\prime}\right] \cdots\left[g_{n-1}, g_{n-1}^{\prime}\right]\right),\left[g_{1}, g_{1}^{\prime}\right]\right.$ $\left.\cdots\left[g_{n}, g_{n}^{\prime}\right]\right)$. But by (3.5) this is $\left(1,\left[g_{1}, g_{1}^{\prime}\right] \cdots\left[g_{n}, g_{n}^{\prime}\right]\right)$.

Corollary 3.7. $[E, E]^{-} \cap A=(1)$.

Proof. By (3.6), if $x \in[E, E]$, then $x=(1, c)$, where $c \in[G, G]$. It follows that $[E, E]^{-} \subseteq\left\{(1, g): g \in[G, G]^{-}\right\}$, and hence $[E, E]^{-} \cap A=(1)$.

\section{Cohomology for symmetric cocycles, the main results}

THEOREM 4.1. Now let $G$ be a second countable locally compact group satisfying *, let $\omega$ be a Borel cocycle $\omega: G \times G \rightarrow A$, and let $(1) \rightarrow A \rightarrow E \stackrel{\pi}{\rightarrow} G \rightarrow$ (1) be the associated central extension. If $\omega$ is symmetric, and if $A$ is second countable and injective for the category of locally compact abelian groups, then $\omega$ is cohomologous to 0 . 
Proof. Let $H=\pi^{-1}\left(H_{1}\right)$, and let $K=\pi^{-1}\left(K_{1}\right)$. Since $G=H_{1} K_{1}$, and since $H_{1}$ and $K_{1}$ are normal in $G$, we have $E=H K$; moreover, $H$ and $K$ are normal in $E$ and closed, since $H_{1}$ and $K_{1}$ are. Because $\omega$ is symmetric and $H_{1}$ and $K_{1}$ are abelian, we know that $H$ and $K$ are abelian by (3.4). Now we have $A \subseteq H \cap K$. Also, by normality, $[K, H] \subseteq H \cap K$, and, since $H$ and $K$ are closed, $[K, H]^{-} \subseteq$ $H \cap K$. By Corollary (3.7), $[K, H]^{-} \cap A=(1)$; therefore $[K, H]^{-} A$ is a direct product, since the factors commute. Now extend the identity map id: $A \rightarrow A$ to a continuous homomorphism $f:[K, H]^{-} \times A \rightarrow A$ by taking $f\left([K, H]^{-}\right)=(1)$. In particular, $f([k, h])=1$ for all $(k, h) \in K \times H$. Since $A$ is an injective, and since $H \cap K$ is abelian, $f$ extends to a continuous homomorphism which we again call $f: H \cap K \rightarrow A$. Now since $H$ and $K$ are abelian, by using injectivity again we can extend this to continuous homomorphisms $f: H \rightarrow A$ and $g: K \rightarrow A$ such that $\left.f\right|_{H \cap K}=\left.g\right|_{H \cap K}$ and $\left.f\right|_{A}=$ id. Moreover, $f\left(k h k^{-1} h^{-1}\right)=1$ for $h \in H$, $k \in K$. But $k h k^{-1} \in H$, and $f$ is a homomorphism of $H$, so that $1=f\left(k h k^{-1} h^{-1}\right)$ $=f\left(k h k^{-1}\right) f(h)^{-1}$. Thus $f\left(k h k^{-1}\right)=f(h)$ for all $k \in K$ and $h \in H$.

We wish to find a continuous homomorphism $\Phi: E \rightarrow A$ such that $\left.\Phi\right|_{H}=f$. Then $\left.\Phi\right|_{H}=\left.f\right|_{A}=$ id, and therefore $A$ would be a direct factor of $E$, so the original sequence would then split, and therefore $\omega$ would be trivial. Define $\Phi$ : $E \rightarrow A$ by $\Phi(h k)=f(h) g(k)$. Now if $h k=h^{\prime} k^{\prime}$, then $h^{\prime-1} h=k^{\prime} k^{-1} \in H \cap K$, so that $f\left(h^{\prime-1} h\right)=g\left(k^{\prime} k^{-1}\right)$. But since $f$ and $g$ are homomorphisms, $f\left(h^{\prime}\right)^{-1} f(h)$ $=g\left(k^{\prime}\right) g(k)^{-1}$, and therefore $f(h) g(k)=f\left(h^{\prime}\right) g\left(k^{\prime}\right)$. It follows that $\Phi$ is well defined. Since this is so, if $h \in H$, then $h=h 1$, where $1 \in K$, and so $\Phi(h)=f(h)$.

Now let $h k$ and $h^{\prime} k^{\prime} \in E$. Then $k h^{\prime} \in k H=H k$, so $k h^{\prime}=h^{\prime \prime} k$. Thus

$$
\begin{aligned}
\Phi\left(h k h^{\prime} k^{\prime}\right) & =\Phi\left(h h^{\prime \prime} k k^{\prime}\right)=f\left(h h^{\prime \prime}\right) g\left(k k^{\prime}\right) \\
& =f(h) f\left(h^{\prime \prime}\right) g(k) g\left(k^{\prime}\right) \\
& =f(h) f\left(k h^{\prime} k^{-1}\right) g(k) g\left(k^{\prime}\right) .
\end{aligned}
$$

On the other hand, $\Phi(h k) \Phi\left(h^{\prime} k^{\prime}\right)=f(h) g(k) f\left(h^{\prime}\right) g\left(k^{\prime}\right)$. Since $A$ is abelian, these are equal, provided that $f\left(k h^{\prime} k^{-1}\right)=f\left(h^{\prime}\right)$ for all $k \in K$ and $h^{\prime} \in H$. Thus $\Phi$ is a homomorphism.

Finally, as for the continuity of $\Phi$, let $H \times K$ act on $E$ as follows: $(h, k) e=$ $h e k^{-1}$. This is a jointly continuous action, and, since $E=H K$, we see that the orbit $\mathcal{O}_{H \times K}(1)=E$, and so the action is transitive. Clearly the stability group $\operatorname{Stab}_{H \times K}(1)=\{(x, x): x \in H \cap K\}$. Since $A$ and $G$ are second countable, $E$ as well as $H \times K$ is second countable, and therefore (see Hochschild [2]), $E$ is $H \times K$ equivariantly homeomorphic to $H \times K / \operatorname{Stab}_{H \times K}(1)$.

Let $h_{n} k_{n}^{-1}$ converge to $h k^{-1}$ in $E$. Then $\left(h_{n}, k_{n}\right) \operatorname{Stab}_{H \times K}(1) \rightarrow$ $(h, k) \operatorname{Stab}_{H \times K}(1)$ in $H \times K /$ Stab. Let $W_{0}$ be a neighborhood of 1 in $A$. Choose a neighborhood $W$ of 1 in $A$ so that $W W^{-1} \subseteq W_{0}$. By continuity of $f$ and $g$, choose neighborhoods $U$ and $V$ in $H$ and $K$, respectively, so that $f(U) \subseteq W$ and 
$g(V) \subseteq W$. Then, for all $n$ large, $(h, k)^{-1}\left(h_{n}, k_{n}\right) \in \operatorname{Stab}_{H \times K}(1) U \times V$; that is, for some $x \in H \cap K,\left(x^{-1} h^{-1} h_{n}, x^{-1} k^{-1} k_{n}\right) \in U \times V$. But then $f\left(x^{-1} h^{-1} h_{n}\right) \in$ $f(U) \subseteq W$ and $g\left(x^{-1} k^{-1} k_{n}\right) \in g(V) \subseteq W$. Since $f$ and $g$ are homomorphisms on $H$ and $K$, respectively, and since $x \in H \cap K$, we have $f(x)^{-1} f(h)^{-1} f\left(h_{n}\right) \in W$ and $g(x)^{-1} g(k)^{-1} g\left(k_{n}\right) \in W$. Because $A$ is abelian,

$$
f(x)^{-1} g(x) f\left(h_{n}\right) g\left(k_{n}\right)^{-1} f(h)^{-1} g(k) \in W W^{-1} \subseteq W_{0} .
$$

But $f$ and $g$ agree on $H \cap K$, so that $\Phi\left(h_{n} k_{n}^{-1}\right) \Phi\left(h k^{-1}\right)^{-1} \in W_{0}$ for $n$ large. Thus $\Phi\left(h_{n} k_{n}^{-1}\right) \rightarrow \Phi\left(h k^{-1}\right)$.

We now come to Theorem (4.2), one of the two main results of our paper, which extends that of [3] to nonabelian groups $G$ satisfying * (as well as to more general groups $A$ ). Theorem (4.2) is analogous to a result of Ritt [8] (see also P. Cassidy [1], especially page 112) which deals with the algebraic but also abelian! case. Then follow some counterexamples to extending. Theorem (4.2) by any method to more general groups. Finally, the other main result, Theorem (4.3), presents the calculation of the cohomology group itself.

THEOREM 4.2. Let $G$ be a connected Lie group satisfying * whose abelianization $G /[G, G]^{-}$is simply connnected, and let $A$ be a second countable injective for the category of locally compact abelian groups. Then the map $B_{A}(G, G)_{a} \rightarrow Z^{2}(G, A)$ $\rightarrow H_{\text {cent }}^{2}(G, A)$ is surjective. In other words any measurable cocycle $\omega$ is cohomologous with a jointly continuous skew symmetric bilinear cocycle.

Proof. Choose $\gamma \in B_{A}(G, G)_{a}$ so that $\gamma^{2}=\omega\left(\omega^{\sim}\right)^{-1}(1.5)$. Then $\omega \gamma^{-1} \gamma=\omega$. If $\omega \gamma^{-1}$ is symmetric, and therefore cohomologous to 1 by (4.1), we would have $\omega^{-\gamma}$. Now since $\gamma^{2}=\omega\left(\omega^{\sim}\right)^{-1}$ we know that $\omega^{\sim \gamma}=\omega \gamma^{-1}$. But $\left(\omega \gamma^{-1}\right)^{-}=\omega \sim$ $\gamma$ since $\gamma$ is skew symmetric.

Remark. Our method also works for abstract groups. It yields the following analogue of Theorem (4.2).

Let $G$ be a group with normal abelian subgroups $H_{1}$ and $K_{1}$ such that $G=H_{1} K_{1}$ and $H_{1} \cap K_{1} \supseteq Z(G)$, and let $A$ be a divisible abelian group. If each element of $G /[G, G]$ has a unique square root, then the map $B_{A}(G, G)_{a} \rightarrow$ $H_{\text {cent }}^{2}(G, A)$ is surjective.

We note that Theorem (4.2) is not true for arbitrary central extensions of Lie groups. For if $A$ does not satisfy the conditions imposed on it by Theorem (4.2), then, even if $G$ satisfies * and $G /[G, G]^{-}$has unique square roots, the conclusion 
of Theorem (4.2) fails in general. For let $G$ be any connected but not simply connected Lie group, let $G^{\sim}$ be its universal covering group, and let $A=\Pi_{1}(G)$, the fundamental group. Then $A$ is a finitely generalized discrete abelian group, and (1) $\rightarrow A \rightarrow G^{\sim} \rightarrow G \rightarrow(1)$ is a central extension of Lie groups. Suppose the corresponding measurable cocycle is equivalent to $\beta \in B_{A}(G, G)$. Then (also by [7]) $\beta$ is jointly continuous. Since $A$ is discrete and $G$ is connected, we see that $\beta\left(g, g^{\prime}\right)=1$ for all $g, g^{\prime}$ so that $G^{\sim}=A \times G$. But since $G^{\sim}$ is connected and $A$ is discrete, we have $A=(1)$ and $G=G^{\sim}$, which is a contradiction. Now choose $G$ satisfying * which is not simply connected, but for which $G /[G, G]$ is simply connected. For example, let $G=N_{r} / \Gamma$, where $\Gamma$ is a discrete central subgroup of $N_{r}$.

Similarly, if $G$ does not satisfy *, then, even if $A$ satisfies the conditions imposed upon it, Theorem (4.2) fails. Let $n \geqslant 2$, let $E=\mathrm{Gl}(n, \mathbb{C})$, let $A=\mathbb{C}^{X} I$, and let $G=\operatorname{SL}(n, C)$. Then $A=Z(E)$ and $E=G A$, so $E / A=G A / A=G / G$ $\cap A=\operatorname{Ad}(G)$, since $G \cap A=Z(G)$, which is a nontrivial finite group. Thus we have a central extension (1) $\rightarrow A \rightarrow E \stackrel{\pi}{\rightarrow} \operatorname{Ad}(G) \rightarrow(1)$ of Lie groups, with $A$ satisfying the conditions of (4.2). If the corresponding cocycle were similar to a bilinear map $\beta$ : $\operatorname{Ad}(G) \times \operatorname{Ad}(G) \rightarrow A$, then for fixed $x \in \operatorname{Ad}(G)$, we have $\beta(x, \operatorname{Ad}(G))=1$ because $\operatorname{Ad}(G)$ is semisimple and $A$ is abelian. Therefore $\beta \equiv 1$, and we can find a continuous cross section $\psi: \operatorname{Ad}(G) \rightarrow E$ to $\pi$. Therefore $\psi(\operatorname{Ad}(G))$ is a semisimple analytic subgroup of $E$ and so is contained in a Levi factor. But since $E$ is reductive it has a unique Levi factor, namely $G$. So $\Psi(\operatorname{Ad}(G)) \subseteq G$. Thus $\Psi$ is a cross section for $\left.\pi\right|_{G}$. Hence $G=Z(G) \times \operatorname{Ad}(G)$, and by connectedness $Z(G)=(1)$. This is impossible since $Z(G)$ is a nontrivial finite group.

Our last step will be to calculate the cohomology itself.

ThEOREM 4.3. Let $G$ be a connected and simply connected Lie group satisfying *, and $A$ be as in (4.2). Then $H_{\mathrm{cent}}^{2}(G, A)=B_{A}(G, G)_{a} / \mathrm{Hom}_{\mathrm{cont}}\left([G, G]^{-}, A\right)$, where the Hom group is identified with a subgroup of $B_{A}(G, G)_{a}$ in the manner described below.

Proof. In view of Theorem (4.2) we know that $B_{A}(G, G)_{a} B^{2}(G, A)=Z^{2}(G, A)$. Hence $H^{2}=Z^{2} / B^{2}=B_{A}(G, G)_{a} B^{2} / B^{2}=B_{A}(G, G)_{a} / B_{A}(G, T)_{a} \cap B^{2}$. It remains to study this intersection. Let $\omega_{f}$ be in the intersection, where $f: G \rightarrow A$ is a measurable function. Since $\omega_{f}$ is skew symmetric, it follows that

(i) $f(g)^{2} f\left(g^{\prime}\right)^{2}=f\left(g g^{\prime}\right) f\left(g^{\prime} g\right)$ for all $g, g^{\prime} \in G$. If $g^{\prime}=z \in Z(G)$, this says that $f(g)^{2} f(z)^{2}=f(g z)^{2}$ for $g \in G, z \in G$. On the other hand, $\omega_{f} \in B_{A}(G, G)$, 
so that for $g, g^{\prime}, g^{\prime \prime} \in G$, we have

(ii) $f(g) f\left(g^{\prime}\right) f\left(g^{\prime \prime}\right) f\left(g g^{\prime} g^{\prime \prime}\right)=f\left(g g^{\prime}\right) f\left(g g^{\prime \prime}\right) f\left(g^{\prime} g^{\prime \prime}\right)$. Taking $g^{\prime}=g$ in (ii) yields $f(g)^{2} f\left(g^{\prime \prime}\right) f\left(g^{2} g^{\prime \prime}\right)=f\left(g^{2}\right) f\left(g g^{\prime \prime}\right)^{2}$, so that, if if $g^{\prime \prime}=z \in Z$, we get, by using (i) and the fact that $A$ is abelian, that $f\left(g^{2} z\right)=f\left(g^{2}\right) f(z)$ for $g \in G$, $z \in Z$. Now each $x \in G$ has a unique square root, so $x=g^{2}$ by Corollary (2.2). Hence $f(x z)=f(x) f(z)$. In particular, $\left.f\right|_{z}$ is a continuous homomorphism and $f(1)=1$. Now by (ii) we get, upon taking $g^{\prime}=g$, that $f(g)^{2} f\left(g^{\prime \prime}\right) f\left(g^{2} g^{\prime \prime}\right)=$ $f\left(g^{2}\right) f^{2}\left(g g^{\prime \prime}\right)$. Multiplying by $f\left(g^{\prime \prime}\right)$, using (i) and cancelling then yields

(iii) $f\left(g^{\prime \prime} g\right) f\left(g^{2} g^{\prime \prime}\right)=f\left(g^{2}\right) f\left(g g^{\prime \prime}\right) f\left(g^{\prime \prime}\right)$. Now $g g^{\prime \prime}=g^{\prime \prime} g z$, where $z \in Z$, since $G$ is 2-step nilpotent, so by the above $f\left(g g^{\prime \prime}\right)=f\left(g^{\prime \prime} g\right) f(z)$. Hence by (iii) $f\left(g^{2} g^{\prime \prime}\right)=f\left(g^{2}\right) f\left(g^{\prime \prime}\right) f(z)$. The fact that each element of $G$ has a square root tells us that

(iv) $f(g h)=f(g) f(h) f\left[g^{1 / 2}, h\right]$ for $g, h \in G$. Thus we see that $\omega_{f}(g, h)=$ $f\left[g^{1 / 2}, h\right]^{-1}$. Now because $G$ is 2-step nilpotent, the map [ , ]: $G \times G \rightarrow[G, G]$ which is obtained by forming commutators is bimultiplicative. From this it follows that $\left[g^{1 / 2}, h\right]=[g, h]^{1 / 2}$, so that

(v) $\omega_{f}(g, h)=f[g, h]^{-1 / 2}$.

In any case $\omega_{f}$ depends only on $\left.f\right|_{\{G, G]}$ rather than on $f$ itself. Now assume only (v). Since $[$,$] is bimultiplicative, and since f$ is a homomorphism on $[G, G]^{-}$, it follows that $\omega_{f}\left(g^{2}, h h^{\prime}\right)=\omega_{f}\left(g^{2}, h\right) \omega_{f}\left(g^{2}, h^{\prime}\right)$. Since any $x=g^{2}$, we see that such an $\omega_{f}$ is multiplicative in the left variable. Also $\left[g, h^{2}\right]=[g, h]^{2}$ and $\left[h, g^{2}\right]=[h, g]^{2}$, so $\omega_{f}\left(g^{2}, h^{2}\right)=f\left[g, h^{2}\right]^{-1}=f[g, h]^{-2}$, while $\omega_{f}\left(h^{2}, g^{2}\right)=$ $f[h, g]^{-2}=f[g, h]^{2}$, so $\omega_{f}\left(h^{2}, g^{2}\right)=\omega_{f}\left(g^{2}, h^{2}\right)^{-1}$. The existence of square roots again tells us that $\omega_{f}$ is skew symmetric, i.e. belongs to $B_{A}(G, G)_{a} \cap B^{2}$. Finally, the map $f \rightarrow \omega_{f}$ from $\operatorname{Hom}_{\text {cont }}\left([G, G]^{-}, A\right) \rightarrow B_{A}(G, G)_{a} \cap B^{2}$ is clearly one-toone since the commutators generate a dense subgroup of $[G, G]^{-}$.

We note that our method also works in the case of abstract groups and yields $H_{\text {cent }}^{2}(G, A)=B_{A}(G, G)_{a} / \operatorname{Hom}([G, G], A)$, where each element of $G$ has a unique square root, $G$ satisfies *, and $A$ is as in the remark following Theorem (4.2).

\section{References}

[1] Phyllis J. Cassidy, 'Unipotent differential algebraic groups', in Contributions to Algebra (a collection of papers dedicated to E. Kolchin), Academic Press (1977), pp. 83-115.

[2] G. Hochschild, The structure of Lie groups (Holden-Day, San Francisco, 1965).

[3] A. Kleppner, 'Multipliers on Abelian groups', Math. Ann. 158 (1965), 11-34.

[4] G. W. Mackey, 'Borel structures in groups and their duals', Trans. Amer. Math. Soc. 85 (1957), 134-165. 
[5] C. C. Moore; 'Extensions and low dimensional cohomology theory of locally compact groups I', Trans. Amer. Math. Soc. 113 (1964), 40-63.

[6] M. Moskowitz, 'Homological algebra in locally compact abelian groups', Trans. Amer. Math. Soc. 127 (1967), 361-409.

[7] M. Moskowitz, 'Uniform boundedness for non-abelian groups', Proc. Cambridge Phil. Soc. 97 (1985), 107-110.

[8] J. F. Ritt, 'Associative differential operations,' Ann. of Math. 52 (1950), 756-765.

\section{Graduate Centre}

City University of New York

33 West 42 Street

New York, New York 10036

U.S.A. 